\title{
Investigations into the mechanism of action of nitrobenzene as a mild dehydrogenating agent under acid-catalysed conditions
}

\author{
M. Lurdes S. Cristiano, ${ }^{a}$ David J. P. Gago, ${ }^{a}$ Antonio M. d'A. Rocha Gonsalves, ${ }^{b}$ \\ Robert A. W. Johnstone, ${ }^{* c}$ Moya McCarron ${ }^{c}$ and Jorge M. T. B. Varejão ${ }^{c, d}$ \\ ${ }^{a}$ FCT, Universidade do Algarve, Campus de Gambelas, Faro 8000, Portugal \\ ${ }^{b}$ Departamento de Quimica, Universidade de Coimbra, Coimbra 3000, Portugal \\ ${ }^{c}$ Department of Chemistry, University of Liverpool, Liverpool, UK L69 3BX \\ ${ }^{d}$ Escola Superior Agraria, IPC, Bencanta, Coimbra 3040, Portugal
}

Received 5th November 2002, Accepted 12th December 2002

First published as an Advance Article on the web 20th January 2003

Protonated nitrobenzene can be used to dehydrogenate a range of hydrocarbons, which already possess at least one double bond. Kinetic and spectroscopic results, together with known electrode potentials, yield approximate limits within which protonated nitrobenzenes can be expected to effect dehydrogenation of hydroaromatic compounds. A high yielding synthesis of benzo[j]fluoranthene is described.

\section{Introduction}

Nitrobenzene and occasionally other nitroarenes have been used for a long time as mild oxidants, which effect dehydrogenation under both acidic and basic conditions. ${ }^{1 a-d}$ Although various reduction products of nitrobenzene have been described as side-products of these dehydrogenations, and there is a general impression that reduction of nitrobenzene with concomitant oxidation of a hydroaromatic to a fully aromatic compound constitutes the "driving force", there is little information on the thermochemistry or mechanism of the reaction and there are no kinetic results. There is no guidance concerning when a particular dehydrogenation might or might not be effected by nitrobenzene and, although there are examples of hydroaromatic systems that did respond, there are reports of some that did not. ${ }^{2}$ Nitrobenzene used under acidic conditions is generally a moderately high temperature process for dehydrogenating hydroaromatic compounds. Dehydrogenation of hydroaromatics under neutral conditions requires very high temperatures and catalysts. ${ }^{3}$ Under neutral or alkaline conditions, nitrobenzene can be used at ambient temperatures for dehydrogenation of thiols, ${ }^{4}$ alkoxides,${ }^{5}$ carbanions, ${ }^{6}$ and radicals, ${ }^{7}$ it also plays a prominent role in SET reactions. ${ }^{8}$

Under acidic or aqueous conditions or with protic solvents, it is unlikely that nitrobenzene would be reduced concomitantly by hydride transfer from a hydroaromatic compound because no evolution of hydrogen has ever been observed. Also, the range of reduction products from nitrobenzene are not those expected for hydride reduction. ${ }^{9}$ Hydride transfer mechanisms have been suggested for some low yielding conversions of chloroxylenes into triarylmethane derivatives but these reactions can be accounted for also by Friedel-Crafts/electron transfer mechanisms. ${ }^{10}$ The role of nitrobenzene in FriedelCrafts reactions has been cogently discussed with the conclusion that electron transfer mechanisms were most likely in many cases. ${ }^{11}$ Hydride type transfer on the surface of a heterogeneous catalyst has been considered as a possibility for reduction of nitro compounds by hydrogen-donors. ${ }^{12}$ In contrast to the hydride type mechanism, reduction of nitrobenzene is well known to take place easily by electron transfer. ${ }^{13}$ Electrochemical and EPR investigations into the reduction of nitrobenzene clearly reveal initial formation of the corresponding anion-radical as the rate determining step, ${ }^{4,14,15}$ This anionradical is then protonated in a second step, which becomes rate determining at high $\mathrm{pH}^{15}$ Whether a nitro compound is reduced to nitroso, azo or azoxy compounds or to hydroxyl- amine, aminophenol or amine depends very much on $\mathrm{pH}$ and temperature, as well as electrode potential. The range of products observed following electron transfer reduction of nitrobenzene is very characteristic of the $\mathrm{pH}$ used.

In the present work, results of dehydrogenation of hydroaromatic compounds with nitroarenes are described, together with other experimental data. There is strong evidence for initial complex formation between protonated nitroarene and the hydroaromatic compound before an electron transfer step and the release of protons that finally leads to dehydrogenation. Analysis of thermochemical data is entirely compatible with such a mechanism.

\section{Results and discussion}

\section{(a) Kinetic experiments}

In the presence of nitrobenzenes, dehydrogenation of 9,10-dihydroanthracene (DHA) under acidic conditions has been found to proceed in very high yield and selectivity to form anthracene, ${ }^{16}$ if acid is omitted, no significant dehydrogenation is observed even at high temperatures or for extended reaction times. Because of the excellent yield and specificity in producing anthracene in this reaction, dehydrogenation of DHA with nitrobenzene was selected as the basis of kinetic experiments in the present work. Trifluoromethanesulfonic acid (TFMSA) was chosen as the acid catalyst because it is strong, non-nucleophilic, non-oxidising and dissolves readily in nitrobenzene.

Initially, it was found that, in neat nitrobenzene as solvent, DHA was oxidised to anthracene at $100{ }^{\circ} \mathrm{C}$ in times varying between 2-3 minutes and an hour, depending on the hydrogen ion concentration. Accordingly, in a first series of kinetic experiments, DHA was dehydrogenated by nitrobenzene to form anthracene, in the presence of various known proportions of TFMSA. The resulting graphs for fractional conversion of DHA into anthracene with time (Fig. 1) show a "burst" phase in that some anthracene appears within a few minutes but this is followed by a steady state phase when anthracene continues to be produced at an almost constant rate. If the molar concentration of acid is increased to be similar to or greater than that of DHA, the burst phase of anthracene formation affords an almost 100\% yield within 1-2 minutes (Fig. 1) and no steady state phase was discernible.

In general terms, the rate of dehydrogenation may be considered to result from an equilibrium constant $(K)$ and the concentrations of reactants. Thus, for protonation of nitrobenzene, 
Table 1 Kinetic data for conversion of 9,10-dihydroanthracene into anthracene in protonated nitrobenzene

\begin{tabular}{|c|c|c|c|c|c|c|c|c|c|c|}
\hline$\left[\mathrm{H}^{+}\right] / \mathrm{mol} \mathrm{dm}^{-3}$ & $T / \mathrm{K}$ & $T_{\text {med }} / \mathrm{K}$ & $k^{\prime} \times 10^{5} / \mathrm{s}^{-1}$ & $1 / T / \mathrm{K}^{-1}$ & Corr $^{a}$ & $E_{\mathrm{a}} / \mathrm{kJ} \mathrm{mol}^{-1}$ & Corr. $^{b}$ & $\Delta H^{*} / \mathrm{kJ} \mathrm{mol}^{-1}$ & $\Delta S^{*} / \mathrm{J} \mathrm{mol}^{-1} \mathrm{~K}^{-1}$ & $\Delta G^{*} / \mathrm{kJ} \mathrm{mol}^{-1}$ \\
\hline \multirow[t]{3}{*}{0.0565} & 353.15 & & 0.5 & 0.00283 & 0.954 & & & & & \\
\hline & 373.15 & 373.15 & 1.8 & 0.00268 & 0.952 & 93.4 & 0.991 & 90.3 & -96.0 & 126.1 \\
\hline & 393.15 & & 11.9 & 0.00254 & 0.987 & & & & & \\
\hline \multirow[t]{3}{*}{0.1130} & 353.15 & & 1.5 & 0.00283 & 0.973 & & & & & \\
\hline & 373.15 & 373.155 & 5.9 & 0.00268 & 0.981 & 87.7 & 0.997 & 84.6 & -101.1 & 122.3 \\
\hline & 393.15 & & 30.5 & 0.00254 & 0.964 & & & & & \\
\hline \multirow[t]{3}{*}{0.1695} & 353.15 & & 3.9 & 0.00283 & 0.995 & & & & & \\
\hline & 373.15 & 373.15 & 14.1 & 0.00268 & 0.971 & 70.3 & 1.000 & 67.2 & -140.5 & 119.6 \\
\hline & 393.15 & & 44.8 & 0.00254 & 0.985 & & & & & \\
\hline \multirow[t]{3}{*}{0.2260} & 353.15 & & 6.5 & 0.00283 & 0.992 & & & & & \\
\hline & 373.15 & 373.15 & 19.5 & 0.00268 & 0.970 & 63.8 & 0.999 & 60.7 & -155.2 & 118.6 \\
\hline & 393.15 & & 59.5 & 0.00254 & 0.991 & & & & & \\
\hline \multirow[t]{3}{*}{0.2825} & 353.15 & & 11.7 & 0.00283 & 0.995 & & & & & \\
\hline & 373.15 & 373.15 & 26.8 & 0.00268 & 0.968 & 56.7 & 0.992 & 53.6 & -171.5 & 117.6 \\
\hline & 393.15 & & 84.2 & 0.00254 & 0.989 & & & & & \\
\hline
\end{tabular}

${ }^{a}$ Correlation coefficients for conversion of DHA to anthracene with time; ${ }^{b}$ Correlation coefficients for $\log \left(k^{\prime}\right)$ and $1 / T$.

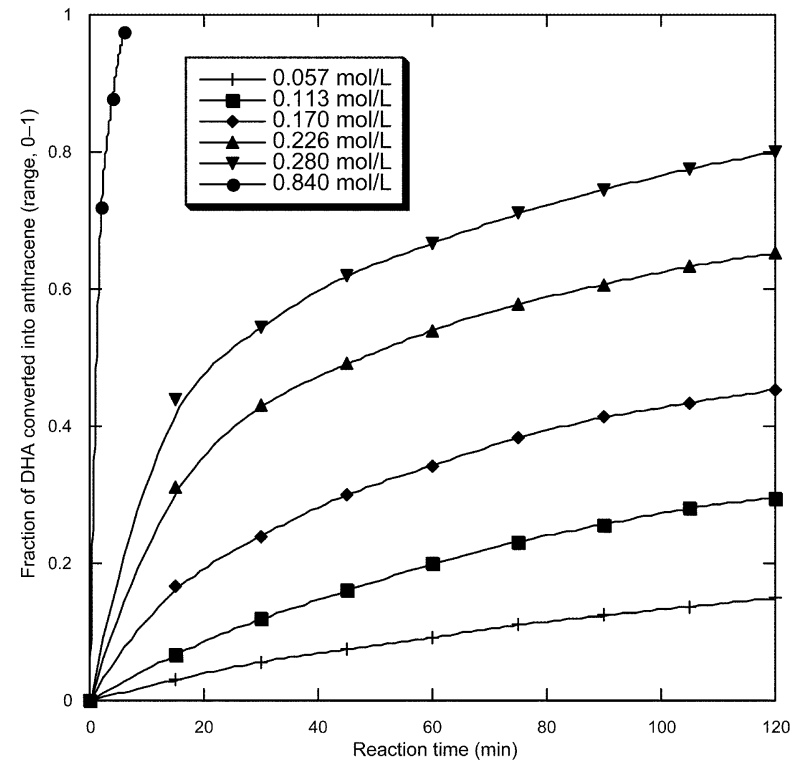

Fig. 1 Conversion of 9,10-dihydroanthracene $(1.1 \mathrm{mmol})$ to anthracene at $100{ }^{\circ} \mathrm{C}$ in nitrobenzene $(2 \mathrm{~mL})$ containing the amounts of trifluoromethanesulfonic acid shown in the figure. For $0.84 \mathrm{~mol} \mathrm{~L}^{-1}$ of acid, the reaction is complete as a burst within about 4 minutes. For $0.057 \mathrm{~mol} \mathrm{~L}^{-1}$ of acid the burst is almost non-existent and a gradual steady formation of anthracene occurs over a period of hours. Other acid concentrations produce mixtures of burst and steady state phases ranging between these extremes.

eqn. (1) affords an expression for $K$, where $\mathrm{PhNO}_{2} / \mathrm{H}^{+}$is a proton-nitrobenzene complex with $n$ undefined.

$$
\begin{aligned}
& \mathrm{PhNO}_{2}+n \mathrm{H}^{+} \leftrightarrow \mathrm{PhNO}_{2} / \mathrm{H}_{n}^{+} ; \\
& K=\left[\mathrm{PhNO}_{2} / \mathrm{H}_{n}{ }^{+}\right] /\left(\left[\mathrm{PhNO}_{2}\right]\left[\mathrm{H}^{+}\right]^{n}\right)
\end{aligned}
$$

A rate of dehydrogenation can be written as in eqn. (2).

Rate of disappearance of DHA =

$$
-d[\mathrm{DHA}] / d \mathrm{t}=k\left[\mathrm{PhNO}_{2} / \mathrm{H}_{n}^{+}\right][\mathrm{DHA}]
$$

By insertion from eqn. (1) and rearranging, eqn. (3) is obtained, in which $\left[\mathrm{PhNO}_{2}\right]$ is considered to be constant because it is used in large excess as solvent.

$$
-d[\mathrm{DHA}] /[\mathrm{DHA}]=k^{\prime} d \mathrm{t}
$$

or, on integration,

$$
\ln [\mathrm{DHA}] /[\mathrm{DHA}]_{0}=-k^{\prime} \cdot \mathrm{t}
$$

Eqn. (3) represents a first order process, in which $k^{\prime}=$ $k \cdot K\left[\mathrm{H}^{+}\right]^{n}\left[\mathrm{PhNO}_{2}\right]$ and $[\mathrm{DHA}]_{0}$ is the concentration of DHA at time zero. For any one hydrogen ion concentration, a plot of $\ln \left([\mathrm{DHA}] /[\mathrm{DHA}]_{0}\right)$ versus $t$ should be linear with a slope equal to $k^{\prime}$. If the hydrogen ion concentration is varied then $k^{\prime}$ should vary in proportion to $\left[\mathrm{H}^{+}\right]^{n}$.

For very short times in the burst phase, $[\mathrm{DHA}] /[\mathrm{DHA}]_{0} \sim$ $1-k^{\prime} \cdot t$ or, $[\mathrm{A}] /[\mathrm{DHA}]_{0}=k^{\prime} \cdot \mathrm{t}=k \cdot K \cdot\left[\mathrm{H}^{+}\right]^{n}\left[\mathrm{PhNO}_{2}\right] t$, in which $[\mathrm{A}]$ is the concentration of anthracene produced in the burst $\left(=[\mathrm{DHA}]_{0}-[\mathrm{DHA}]\right)$. For the initial burst phase, a plot of $\ln \left([\mathrm{A}] /[\mathrm{DHA}]_{0}\right)$ versus $\ln \left[\mathrm{H}^{+}\right]$should then be a straight line of slope $n$. When this was done from the graphs in Fig. 1, the value of $n$ was found to be close to 2 . No attempt was made to obtain a more accurate value because accurate ones for $n$ were later extracted from the full rate measurements described below. However, the value does serve to indicate that TFMSA appears to doubly protonate nitrobenzene. The result is in keeping with UV/visible measurements on complex formation between protonated nitrobenzene and DHA (discussed below).

In the main second series of kinetic experiments, the initial rates of the burst phase for oxidation of DHA were found to obey a simple first order plot, as expected from the above analysis. In all the following experiments, the initial concentration of DHA was the same. For each of three temperatures $(80,100$, $120{ }^{\circ} \mathrm{C}$ ), the rate of formation of anthracene was monitored with time and, by application of eqn. (3), a value for $k^{\prime}$ was obtained at each hydrogen ion concentration (Table 1). For each value of $\left[\mathrm{H}^{+}\right]$, a plot of $\ln \left(k^{\prime}\right)$ versus $1 / T$ yielded the Arrhenius activation energy, and the enthalpy, entropy and the free energy of activation (Table 1).

The enthalpy of activation falls by nearly $40 \mathrm{~kJ}^{\mathrm{mol}}{ }^{-1}$ as the acidity of the medium increases five-fold (Table 1). At the same time, the entropy of activation becomes more negative by almost $80 \mathrm{~J} \mathrm{~K}^{-1} \mathrm{~mol}^{-1}$. The combined enthalpies and entropies offset each other such that the overall free energy change varies by only $9 \mathrm{~kJ} \mathrm{~mol}^{-1}$ over a $40^{\circ}$ range in temperature and the fivefold increase in acidity. The changes imply an activation energy barrier that becomes thermally easier to cross with increase in acidity but a transition state that becomes more difficult to form.

To examine the effect of acidity on $k^{\prime}, \ln \left(k^{\prime}\right)$ was plotted against $\ln \left[\mathrm{H}^{+}\right]$for each temperature. These graph plots were straight lines with slopes $(n)$ equal to $2.0\left(80^{\circ} \mathrm{C}\right), 1.72\left(100^{\circ} \mathrm{C}\right)$ and $1.18\left(120^{\circ} \mathrm{C}\right)$. New rate constants $\left(k^{\prime \prime}\right)$ were calculated from the expression, $k^{\prime \prime}=k^{\prime} /\left[\mathrm{H}^{+}\right]^{n}$ (Table 2). At any one temperature, the rate constants $\left(k^{\prime \prime}\right)$ are almost equal within experimental error. Since $[1]^{n}=1$, the new $k^{\prime \prime}$ values are essentially just $k^{\prime}$ for a uniform hydrogen ion concentration of 1 mol. $\mathrm{L}^{-1}$. The $k^{\prime \prime}$ values were used in a second series of Arrhenius plots of $\ln \left(k^{\prime \prime}\right)$ against the reciprocal of absolute temperature to give new 
Table 2 Kinetic data for conversion of 9,10-dihydroanthracene into anthracene changed to a uniform acid concentration, $\left[\mathrm{H}^{+}\right]=1$ mol $\mathrm{L}^{-1}$

\begin{tabular}{|c|c|c|c|c|c|c|c|c|c|c|}
\hline$\left[\mathrm{H}^{+}\right] / \mathrm{mol} \mathrm{dm}^{-3}$ & $T / \mathrm{K}$ & $T_{\text {med }} / \mathrm{K}$ & $\begin{array}{l}k^{\prime \prime} \times 103 / \\
\mathrm{s}^{-1 b}\end{array}$ & $\ln \left(k^{\prime \prime}\right)$ & $1 / T / \mathrm{K}^{-1}$ & Corr. $^{a}$ & $\begin{array}{l}E A / \\
\mathrm{kJ} \mathrm{mol}^{-1}\end{array}$ & $\begin{array}{l}\Delta H^{* /} \\
\mathrm{kJ} \mathrm{mol}^{-1}\end{array}$ & $\begin{array}{l}\Delta S^{* /} \\
\mathrm{kJ} \mathrm{mol}^{-1} \mathrm{~K}^{-1}\end{array}$ & $\begin{array}{l}\Delta G^{* /} \\
\mathrm{kJ} \mathrm{mol}^{-1}\end{array}$ \\
\hline \multirow[t]{3}{*}{0.057} & 353.15 & & 1.47 & -6.5208 & 0.00283 & & & & & \\
\hline & 373.15 & 373.15 & 2.48 & -6.0011 & 0.00268 & 0.997 & 25.2 & 22.1 & -237.5 & 110.7 \\
\hline & 393.15 & & 3.52 & -5.6497 & 0.00254 & & & & & \\
\hline \multirow[t]{3}{*}{0.113} & 353.15 & & 1.15 & -6.7675 & 0.00283 & & & & & \\
\hline & 373.15 & 373.15 & 2.51 & -5.9864 & 0.00268 & 0.985 & 33.3 & 30.2 & -215.6 & 110.7 \\
\hline & 393.15 & & 3.63 & -5.6188 & 0.00254 & & & & & \\
\hline \multirow[t]{3}{*}{0.170} & 353.15 & & 1.38 & -6.5863 & 0.00283 & & & & & \\
\hline & 373.15 & 373.15 & 2.99 & -5.8114 & 0.00268 & 0.954 & 28.2 & 25.1 & -227.9 & 110.1 \\
\hline & 393.15 & & 3.63 & -5.6188 & 0.00254 & & & & & \\
\hline \multirow[t]{3}{*}{0.226} & 353.15 & & 1.29 & -6.6562 & 0.00283 & & & & & \\
\hline & 373.15 & 373.15 & 2.52 & -5.9824 & 0.00268 & 0.984 & 28.5 & 25.4 & -228.5 & 110.7 \\
\hline & 393.15 & & 3.43 & -5.6741 & 0.00254 & & & & & \\
\hline \multirow[t]{4}{*}{0.283} & 353.15 & & 1.48 & -6.5175 & 0.00283 & & & & & \\
\hline & 373.15 & 373.15 & 2.36 & -6.0485 & 0.00268 & 1.000 & 26.8 & 23.6 & -233.7 & 110.9 \\
\hline & 393.15 & & 3.74 & -5.5899 & 0.00254 & & & & & \\
\hline & & & & & & Mean values = & 28.4 & 25.2 & -228.6 & 110.6 \\
\hline
\end{tabular}

values for $E_{\mathrm{a}}, \Delta H^{*}, \Delta S^{*}$ and $\Delta G^{*}$ for reaction at $\left[\mathrm{H}^{+}\right]=1 \mathrm{~mol}$ $\mathrm{L}^{-1}$. Once the acidity term is made uniform, the enthalpy and entropy terms also become uniform (Table 2).

The new values of $E_{\mathrm{a}}, \Delta H^{*}, \Delta S^{*}$ and $\Delta G^{*}$ for $\left[\mathrm{H}^{+}\right]=1$ allow some deductions to be made. First, the free energy of activation is constant. Second, the activation energy for the burst phase is quite low and is commensurate with the sorts of values found for electron transfer in simple redox reactions. ${ }^{17}$ Third, the entropic factor is quite large and negative. In terms of randomness in the transition state, this result means that it becomes increasingly difficult for the reaction to proceed as temperature rises. The power of the hydrogen ion concentration decreases with increasing temperature as would be expected for a complex of nitrobenzene and $\mathrm{H}^{+}$that increasingly dissociates as the temperature rises. The enthalpy and entropy are in accord with the concept of an initial complex formed from DHA, nitrobenzene and protons, which decomposes easily by a mechanism that has very low enthalpy of activation, similar to the requirements of simple redox reactions. The complex becomes increasingly difficult to form as temperature increases because of increasing translational and rotational motion of the components. The rapid burst phase of release of anthracene early in the reaction is characterised by an activation energy that is small at high acidities, but which increases with either or both decreasing acidity and increasing temperature.

These kinetic results from DHA are next compared with information gleaned from other experiments described below or from thermochemical constants and other data found in the literature.

\section{(b) Heat of reaction}

Having obtained the activation energy, it becomes of interest to obtain the heat of reaction $\left(\Delta H_{\mathrm{R}}\right)$ for the conversion of DHA and nitrobenzene into anthracene and aniline, the end products. It seems to be generally believed that the "driving force" for these sorts of dehydrogenations results from the energy gained by converting nitrobenzene to aniline and by the "aromatization" process of DHA being converted into anthracene (A reaction 4). ${ }^{1 a, b, 16}$

$$
3 \mathrm{DHA}+\mathrm{PhNO}_{2} \longrightarrow 3 \mathrm{~A}+\mathrm{PhNH}_{2}+2 \mathrm{H}_{2} \mathrm{O}
$$

The heats of formation of all these components are known. ${ }^{18}$ From these values and eqn. (4), it is easily calculated that, if water is neglected, the reaction would be expected to be endothermic but inclusion of water makes the reaction exothermic

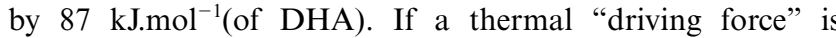
considered, it is clearly due to the formation of water in the reduction of nitrobenzene to aniline and not to the "aromatization" process. By postulates relating exo- and endothermicities of reactions to their activation energies, ${ }^{21}$ it is clear that exothermic dehydrogenation implies a structure of the transition state resembling more the starting materials than it does the products of reaction, viz., the transition state for this dehydrogenation resembles the initial complex, DHA- $\mathrm{PhNO}_{2}-\mathrm{H}^{+}$. Once reaction is complete, return to the starting materials is unlikely because of the high energy demand required to offset the heat of formation of water.

\section{(c) The complex of DHA-PhNO$-\mathrm{H}^{+}$}

When DHA is added to a solution of TFMSA in $\mathrm{PhNO}_{2}$, a yellow colour develops at room temperature. If the acidity is increased, the colour deepens and becomes orange-brown at the highest acid concentrations used in these experiments. On examination of the $\mathrm{UV} /$ visible spectra of $\mathrm{DHA}-\mathrm{PhNO}_{2}-\mathrm{H}^{+}$ mixtures, it is found that, as acid strength is increased, a new charge transfer band ${ }^{22}$ begins to appear at about $470 \mathrm{~nm}$. In a standard process for examining complexes in solution, ${ }^{23 a}$ $\log ($ absorption at $520 \mathrm{~nm})$ was plotted against $\log \left[\mathrm{H}^{+}\right]$. A straight line was obtained of slope equal to 1.81 . The result indicates that, over the hydrogen ion concentration considered, a complex is formed between protonated nitrobenzene and DHA, in which the proton concentration is effective to a power value close to 2 , which accords with the results of the kinetic experiments described above. Other methods have been used to examine room temperature complexes of nitrobenzene with acids and have produced relationships in which the nitrobenzene/proton number varies from $2: 1$ to $1: 2,{ }^{24}$ and a value of $1: 1: 1$ has been reported for a complex of a Lewis acid $\left(\mathrm{AlCl}_{3}\right)$ with $\mathrm{PhNO}_{2}$ and anthracene. ${ }^{25}$

\section{(d) Electron transfer from DHA to protonated nitrobenzene}

The basic electron transfer reaction (eqn. (5)) can be viewed as two half reactions, for which electrochemical reduction and oxidation potentials are known.

$$
\begin{gathered}
\mathrm{DHA}^{+}+\mathrm{e}^{-} \rightarrow \mathrm{DHA} \\
\mathrm{PhNO}_{2}+\mathrm{e}^{-} \rightarrow \mathrm{PhNO}_{2}^{-}(1) \\
\hline \mathrm{DHA}+\mathrm{PhNO}_{2} \rightarrow \mathrm{DHA}^{+}+\mathrm{PhNO}_{2}^{-}(2) \\
\Delta H_{\mathrm{R}}=E_{1 / 2}(2)-E_{1 / 2}(1)
\end{gathered}
$$

The values for $E_{1 / 2}(1)$ and $E_{1 / 2}(2)$ are 1.87 and $-0.56 \mathrm{~V}$ respectively, ${ }^{26}$ indicating an overall energy requirement of $-2.43 \mathrm{~V}\left(233 \mathrm{~kJ} \mathrm{~mol}^{-1}\right)$ at $\mathrm{pH}$ 7.0. This requirement is endothermic and far greater than the observed kinetic activation 
energy of, for example $70.3 \mathrm{~kJ} \mathrm{~mol}^{-1}$ at $\left[\mathrm{H}^{+}\right]=0.17 \mathrm{~mol} \mathrm{~L}^{-1}$ (Table 1). There are other factors that must be taken into account in estimating $\Delta H_{\mathrm{R}}$ of eqn. (5). The first of these is the change in $E_{1 / 2}(2)$ as $\mathrm{PhNO}_{2}$ is acidified. Two separate reports yield information on the magnitude of $E_{1 / 2}(2)$ with change in $\mathrm{pH} .{ }^{27}$ If the two sets of data are plotted on the same graph, a linear correlation is observed, from which the reduction potential for nitrobenzene can be represented by the equation, $E_{1 / 2}(2)$ $=-0.14-0.06[\mathrm{pH}]$ volts (correlation coefficient $=0.984$ ). The slope is close to the expected value of 0.059 expected for single electron transfer. ${ }^{28}$ For example, at a hydrogen ion concentration of $0.85 \mathrm{~mol} \mathrm{~L}^{-1}$, as used in some of the experiments described here, the $\mathrm{pH}$ of 0.07 gives a value for $E_{1 / 2}(2)$ of $-0.14 \mathrm{~V}$. This new value decreases the overall energy requirement from $2.43 \mathrm{~V}$ to $2.01 \mathrm{~V}\left(193 \mathrm{~kJ} \mathrm{~mol}^{-1}\right)$. Thus, the effect of acidifying a mixture of DHA and nitrobenzene to $\mathrm{pH} 0.07$ is to reduce the energy requirement for electron transfer by $40 \mathrm{~kJ}$ $\mathrm{mol}^{-1}$ (Fig. 2). However, at $193 \mathrm{~kJ} \mathrm{~mol}^{-1}$, the process still appears to be very endothermic and substantially greater than the thermal component of the activation energy (about 60 $100 \mathrm{~kJ} \mathrm{~mol}^{-1}$ depending on acidity; Table 1).

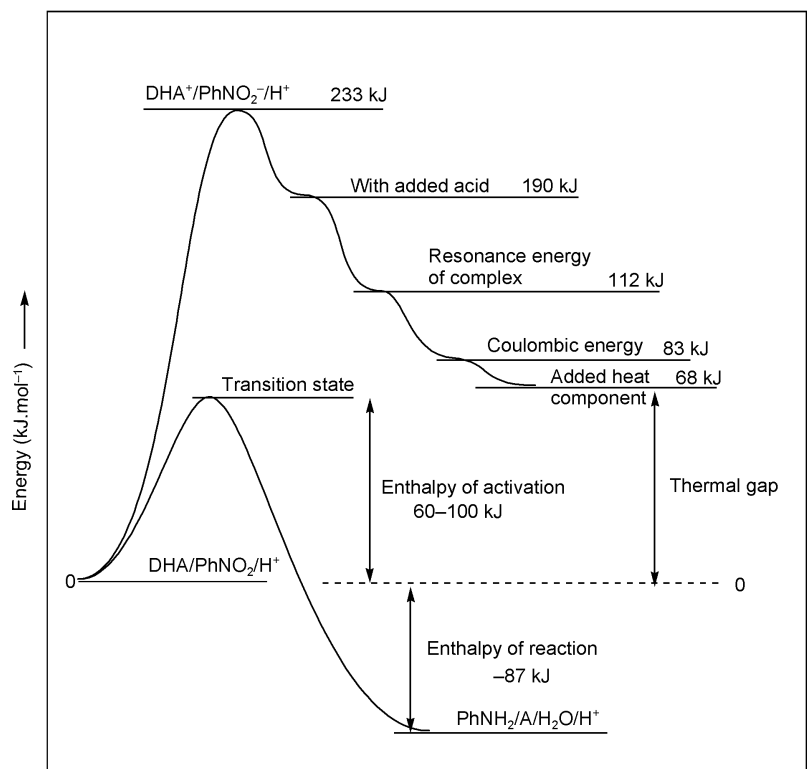

Fig. 2 An outline of the various energies discussed in the text. The thermal gap represents the difference in energy between the starting materials $\left(0 \mathrm{~kJ} \mathrm{~mol}^{-1}\right)$ and the same materials after mixing and allowing for acidity effects, complex formation, Coulombic energy and added heat energy in raising the temperature of the mixture to $100{ }^{\circ} \mathrm{C}$. The thermal gap is of a similar magnitude to the observed enthalpy of activation, shown here bracketed over a range of acidities.

\section{(e) Energetics of complex formation between protonated $\mathrm{PhNO}_{2}$ and DHA}

The appearance of a new charge-transfer band at $470 \mathrm{~nm}$ on acidifying a solution of DHA in nitrobenzene with TFMSA indicates the formation of a stable entity, in which an electron is already partially transferred from DHA to $\mathrm{PhNO}_{2}$. This sort of charge transfer complex formation has been well researched in the past. ${ }^{23 b, 29}$ The stability of the complex can be estimated from three major factors: the ionization energy of DHA, the electron affinity of protonated $\mathrm{PhNO}_{2}$ and a Coulombic term resulting from the charges arising when an electron is transferred. ${ }^{30}$ From these values, a resonance energy can be calculated for the complex. As shown, ${ }^{30}$ the resonance energy is calculated to be $0.81 \mathrm{eV}\left(78 \mathrm{~kJ} \mathrm{~mol}^{-1}\right)$ and the Coulombic energy $0.3 \mathrm{eV}\left(29 \mathrm{~kJ} \mathrm{~mol}^{-1}\right){ }^{33}$ The calculated resonance energy for the DHA complex may at first appear to be high but it should be noted that a $1: 2$ complex of DHA with 1,3,5-trinitrobenzene has been prepared and is sufficiently stable for its structure to have been determined by X-ray diffraction methods. The resulting crystal structure showed that each benzene ring of each DHA molecule independently formed a complex with an acceptor molecule (1,3,5-trinitrobenzene) thereby giving a $1: 2$ complex for the DHA. The interaction between each DHA and the two acceptor molecules was sufficiently strong as to cause a shape change in the DHA. ${ }^{38}$ If there are effectively two separate complexation sites in the same molecule, the total resonance energy is approximately doubled. Good acceptors such as tetracyanoethylene having a similar electron affinity to that of protonated nitrobenzene form $1: 1$ complexes with even simple aromatic $\pi$-donors such as hexamethylbenzene and these complexes have stabilities of about $30 \mathrm{~kJ} \mathrm{~mol}^{-1} \cdot{ }^{39}$ These figures indicate that a resonance energy of $78 \mathrm{~kJ} \mathrm{~mol}^{-1}$ is reasonable for a strong double complex of DHA with protonated nitrobenzene.

Together, the resonance and Coulombic energy terms reduce the overall requirement for transfer of an electron from DHA to protonated $\mathrm{PhNO}_{2}$ from 193 to $86 \mathrm{~kJ} \mathrm{~mol}^{-1}$ (Fig. 2). Since the reaction is carried out at $100^{\circ} \mathrm{C}$, there is an additional heat component of about $15 \mathrm{~kJ} \mathrm{~mol}^{-1}$, ${ }^{40}$ bringing the total energy requirement for electron transfer to $63 \mathrm{~kJ} \mathrm{~mol}^{-1}$. This estimated value for the heat energy needed to allow electron transfer from DHA to protonated nitrobenzene is similar to the enthalpies of activation found in the kinetic experiments (Fig. 2). This close similarity between the enthalpy for an electron-transfer process and the observed enthalpy of activation increases the probability that the mechanism starts with formation of a strong complex between DHA and protonated benzene (a charge-transfer complex). Heating to $100{ }^{\circ} \mathrm{C}$ effects complete transfer of the electron. As shown in electrochemical work on $\mathrm{PhNO}_{2}$, once initial electron-proton transfer has occurred at the reduction potential, a further cascade of electrons and protons completes the reduction to aniline at the same potential. ${ }^{15}$

(f) The importance of the ionization energy of the substance to be dehydrogenated

If the "driving force" for the reaction is due to the ease of transfer of an electron from the substance to be dehydrogenated (DHA in this case) and the electron affinity of protonated nitrobenzene then it is clear that the ionization energy $(I)$ of the hydrogen donor must play an important role. For all dehydrogenations involving protonated nitrobenzene, the electron affinity of the protonated nitrobenzene $(E A)$ remains constant for any one hydrogen ion concentration. ${ }^{32}$ The Coulombic energy is small and may be neglected in this discussion. Therefore, the energy requirement for electron transfer can be approximated to the difference in the energy $(I)$ needed to ionize DHA and the energy $(E A)$ gained, viz., $I-E A$. It is now possible to consider what effect a change in ionization energy would have on the rate of dehydrogenation. The ionization energy $(I)$ of the substrate to be dehydrogenated is important in determining whether or not an initial charge transfer complex will be formed with sufficient gain in energy as to offset the notional energy requirement for complete transfer of an electron in the absence of complex formation.

For the hydrobenzene compounds, cyclohexane, cyclohexene and cyclohexadiene, the ionization energies are 9.86, 8.95, and $8.40 \mathrm{eV}$ respectively. ${ }^{41}$ In the present work, attempts were made to dehydrogenate these compounds under similar condition to those used for the kinetic experiments with DHA. There were three quite different results. At $50{ }^{\circ} \mathrm{C}$, cyclohexane was not dehydrogenated, even on extended heating. Cyclohexene underwent slow conversion to benzene at $50{ }^{\circ} \mathrm{C}(40 \%$ in one hour). In marked contrast, cyclohexa-1,3-diene itself and three other cyclohexadienes ( $\alpha$-terpinene, $4(R)$ - and $4(S)$-isopropenylmethylcyclohex-1-ene) underwent rapid dehydrogenation to benzene or substituted benzenes at $22{ }^{\circ} \mathrm{C}$ within about 1 minute. These last materials are all excellent hydrogen donors 
in catalysed heterogeneous transfer hydrogenation. ${ }^{12}$ In changing from an ionization energy of $8.4 \mathrm{eV}$ to $8.95 \mathrm{eV}$ and then to $9.86 \mathrm{eV}$, dehydrogenation changes from being very easy (cyclohexadiene) to being unobservable (cyclohexane) under the reaction conditions used here. Cyclohexene is intermediate in behaviour, undergoing slow dehydrogenation. The behaviour is in keeping with the strengths of complexes formed between such compounds and electron acceptors. ${ }^{22}$ In practical terms, an ionization energy of about 8.9 to $9.0 \mathrm{eV}$ (or $I-E=6.3-6.4$ $\mathrm{eV}$ ) can be regarded as an upper limit for deciding whether or not dehydrogenation is likely to occur with protonated nitrobenzene at modest temperatures.

In other experiments described below, 1,2,3,4-tetrahydronaphthalene (tetralin; an "analogue" of cyclohexane) gave very small amounts of naphthalene and resisted attempts to dehydrogenate it even at $150{ }^{\circ} \mathrm{C}$ and high acidity. However, GC/ MS revealed some formation of two isomers with $M_{\mathrm{r}}=262$ resulting from acid-catalysed condensation of two molecules of tetralin: these were not investigated further as they were not products of dehydrogenation. In contrast, 1,2-dihydronaphthalene (dialin; an analogue of cyclohexene) was readily dehydrogenated at $150{ }^{\circ} \mathrm{C}$ to give some naphthalene but there were added complications from acid-induced reactions; the major product was benzo[j]fluoranthene.

Dehydrogenation of 9,10-dihydrophenanthrene was slow even at $100{ }^{\circ} \mathrm{C}$. Toluene and 1,2-dimethylbenzene did not dehydrogenate at $100{ }^{\circ} \mathrm{C}$. Perhydroaromatic compounds seem to resist dehydrogenation by nitrobenzene, as would be expected from a mechanism involving electron transfer in an initially formed complex of substrate and nitrobenzene because the ionization energy for an alkane is much greater than that for an alkene. Examples, from the literature support this proposal: 1,2,3,4,5,7,8,8a,9,10,10a-dodecahydrophenanthrene dicarboxylic acid anhydride could not be dehydrogenated by nitrobenzene ${ }^{2}$ and dibenzyl with nitrobenzene could only be converted into stilbene in modest yield even at $245{ }^{\circ} \mathrm{C}$, under pressure and over a period of 16 hours in the presence of a palladium catalyst. ${ }^{3 a}$

\section{(g) Ionization energy and the dehydrogenation of anions}

There are many instances of the ease with which anions and radicals may be dehydrogenated with neutral or alkaline nitrobenzene. In these cases, the concentrations of hydrogen ion are very low and the energy gain of $40 \mathrm{~kJ} \mathrm{~mol}^{-1}$ from protonation of nitrobenzene cannot apply. However, for anions, their ionisation energies are simply the negative values of the electron affinities of the corresponding radicals. Electron affinities for radicals are generally of the order of $1-2 \mathrm{eV}$ and therefore, for an anion, the ionization term $(I)$ in complex formation ${ }^{30}$ is only about $1-2 \mathrm{eV}$, which is about $6-7 \mathrm{eV}$ below the ionization energy even of an easily dehydrogenated cyclohexadiene. The electron affinity of non-protonated nitrobenzene is $1.01 \mathrm{eV}^{35}$ Thus, $I-E A$ for dehydrogenation of anions is only about 0-2 eV compared with the $6.3-6.4 \mathrm{eV}$ needed to dehydrogenate alkenes. Complex formation between nitrobenzene and an anion should then be expected to be very easy and provide more than enough energy to offset the loss of electron affinity entailed in changing to unprotonated nitrobenzene. ${ }^{38}$ Oxidation of anions by nitrobenzene can be expected to be easy. This ease of dehydrogenation has been found for SET reactions, and in the easy oxidations of thiolates, ascorbates and carbanions of various kinds. ${ }^{4-9}$ Dehydrogenation of all such entities has been examined by EPR and been shown to proceed through initial complex formation with nitrobenzene, followed by electron transfer to form the anion-radical of nitrobenzene. Similar reactions have been investigated in cellular systems, in which various drugs containing nitro groups have been shown by EPR to react by initial transfer of an electron to the nitro group. ${ }^{6 b}$ (h) The effect of a change in reduction potential through the use of other nitroarenes

The above discussion has shown that the effect of protonation of nitrobenzene is to lead to an increase in its electron affinity (a decrease in its reduction potential), which in turn leads to a concomitant ease of use of nitrobenzene as an oxidant. However, the electron affinity of nitroarenes can also be changed considerably by varying the substituents in the aromatic ring. Thus, for a series of 4-substituted nitrobenzenes, electron affinity varies from $2.0 \mathrm{eV}$ for 1,4-dinitrobenzene to $0.91 \mathrm{eV}$ for 4-methoxynitrobenzene. From the equation, $E A=1.49\left(E_{1 / 2}\right)+$ $2.79,{ }^{32}$ this difference in $E A$ s translates into a change in $E_{1 / 2}$ of $0.73 \mathrm{~V}$, viz., it becomes much more difficult to reduce the nitro group in 4-methoxynitrobenzene than it does in 1,4-dinitro benzene. These changes should make 1,4-dinitrobenzene a better oxidant ( $I-E A$ is smaller) than nitrobenzene and 4-methoxynitrobenzene a worse oxidant ( $I-E A$ is larger). Electron affinities correlate linearly with reduction potentials $\left(E_{1 / 2}\right){ }^{32}$ Also, reduction potentials of substituted nitrobenzenes in DMSO have been found to correlate with $\sigma_{\mathrm{p}}{ }^{-}$values. ${ }^{42}$ When electron affinities for $p$-substituted nitrobenzenes were plotted against $\sigma_{\mathrm{p}}{ }^{-}$constants, ${ }^{43}$ a linear correlation was found with $E A$ $=0.69\left(\sigma_{p}{ }^{-}\right)+1.09 \mathrm{eV}$. Therefore, logarithms of rate constants for dehydrogenation of substrates using $p$-substituted nitrobenzenes should vary linearly with $\sigma_{\mathrm{p}}{ }^{-}$constants for the substituents $\left(I-E A=I-0.69 \sigma_{\mathrm{p}}{ }^{-}+1.09\right)$. This effect of change of substituent was examined by dehydrogenation of a porphyrinogen with different nitrobenzenes. Nitrobenzene in acetic acid is a good oxidant for converting various porphyrinogens (made from substituted benzaldehydes and pyrrole) into the porphyrin itself. ${ }^{44}$ The reaction has been optimised ${ }^{44}$ but it is sometimes accompanied by significant quantities of the corresponding chlorins, in which two of the hydrogens in the porphyrin have not been removed. Nitrobenzene in acetic acid is not a sufficiently powerful oxidant as to convert at least some chlorins (3; Scheme 1) into porphyrins.

In the present work, solutions of 5,10,15,20-tetrakis(4-methoxyphenyl)porphyrinogen (2; Scheme 1) were prepared

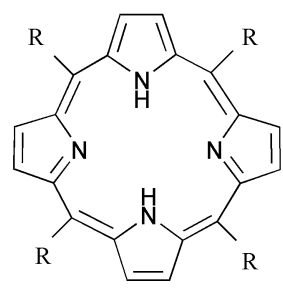

1; R = 4-methoxyphenyl

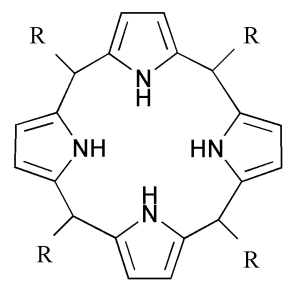

$2 ; \mathrm{R}=4$-methoxyphenyl

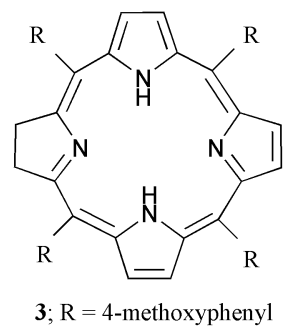

Scheme 1

at room temperature by reducing solutions of $5,10,15,20$ tetrakis(4-methoxyphenyl)porphyrin (1; Scheme 1) in acetic acid with zinc dust. None of the chlorin (3; Scheme 1) was observed in these experiments. As expected, solutions of the porphyrinogen were colourless and showed no UV/visible absorption at the usual porphyrin Soret band wavelength near $420 \mathrm{~nm}$. The porphyrinogen was dehydrogenated by adding a solution of it to a solution of a $p$-substituted nitrobenzene in acetic acid at $90{ }^{\circ} \mathrm{C}$. The appearance of the Soret band representing the reformation 
of porphyrin was monitored by UV/visible measurements. Initial rate constants were obtained for the first few minutes of reaction by recording the visible absorption at the Soret wavelength with time. When the logarithms of these first order rate constants are plotted against $\sigma_{\mathrm{p}}{ }^{-}$constants ${ }^{43}$ for several substituted nitrobenzenes, an approximately linear correlation is observed. The nitroarene with the largest ${\sigma_{\mathrm{p}}}^{-}$value $(1,4-\mathrm{di}-$ nitrobenzene) was found to increase the rate of dehydrogenation 10 -fold compared with the rate for the nitro compounds with the smallest $\sigma_{\mathrm{p}}{ }^{-}$(4-methoxynitrobenzene; Fig. 3). The

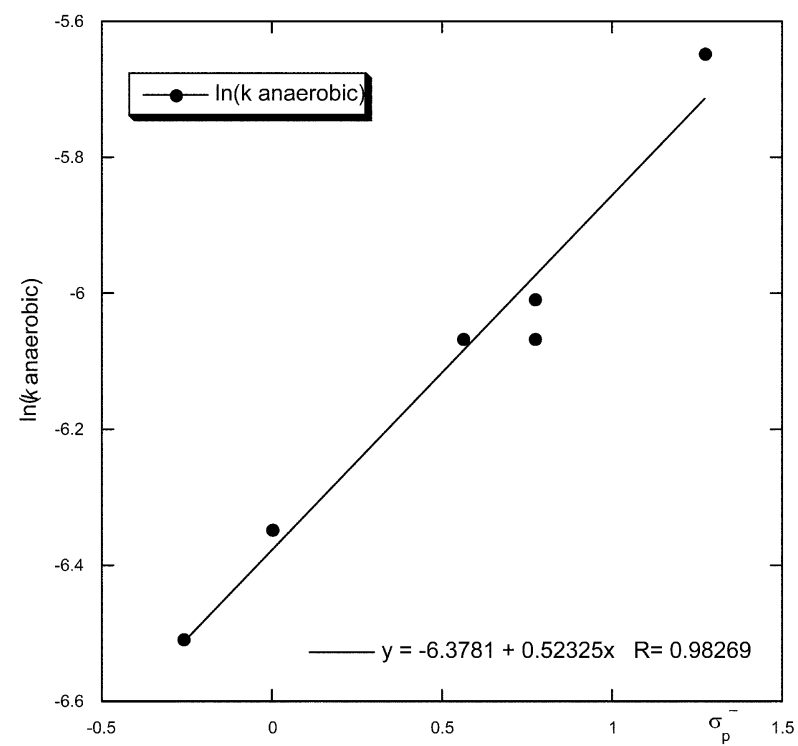

Fig. 3 Logarithms of the initial rate of formation of 5,10,15,20tetrakis(4-methoxyphenyl)porphyrin $(2 ;$ Scheme 1$)$ from the corresponding porphyrinogen $(\mathbf{1}$; Scheme 1$)$ in the presence of a nitrobenzene. Seven $p$-substituted nitrobenzenes, $\mathrm{RC}_{6} \mathrm{H}_{4} \mathrm{NO}_{2}$, were used with $\sigma_{\mathrm{p}}{ }^{-}$values as follows: $\mathrm{NO}_{2}(1.27), \mathrm{H}(0), \mathrm{NH}_{3}{ }^{+}(0.56), \mathrm{N}\left(\mathrm{CH}_{3}\right)_{3}{ }^{+}$ $(0.77), \mathrm{CH}_{3} \mathrm{O}(-0.26), \mathrm{CO}_{2} \mathrm{H}(0.77)$, all from reference 43 .

slope of the graph in Fig. $3(0.52)$ is very similar to the slope of the correlation between $E A$ and $\sigma_{\mathrm{p}}{ }^{-}(0.69)$. Therefore, the rate of dehydrogenation of porphyrinogen to porphyrin does vary with the reduction potential of the substituted nitrobenzene used as oxidant.

\section{(i) Dehydrogenation of 1,2-dihydronaphthalene by nitrobenzene}

Cyclohexane, cyclohexene, cyclohexadienes and tetralin have already been commented on but the dehydrogenation of 1,2dihydronaphthalene needs further comment. Under the usual conditions with protonated nitrobenzene at $100{ }^{\circ} \mathrm{C}$, the starting material disappeared quickly but little dehydrogenation to naphthalene was observed; a similar small quantity of tetralin was formed, the naphthalene and tetralin presumably being formed by disproportionation. However, two major higher boiling components were found. By GC/MS, these had molecular masses of 262 and 258 and appear to be formed from an acidcatalysed dimerization to mass 260 , followed by simple disproportionation to 262 and 258 . With continued heating at $150{ }^{\circ} \mathrm{C}$, the yield of the dimers went down and a third major component appeared. This was isolated from the reaction mixture as a crystalline solid and found to be benzo[j]fluoranthene. Further acid-catalysed ring closure of the initial dimers followed by dehydrogenation is the most likely mechanism for this reaction (Scheme 2). Both benzo[ $j]$ fluoranthenes and benzo $[k]$ fluoranthenes have been prepared as a mixture in a Scholl type synthesis by oxidising 1,2'-binaphthylene over $\mathrm{CrO}_{3}-\mathrm{Al}_{2} \mathrm{O}_{3}$ at $495-500{ }^{\circ} \mathrm{C}^{45 a}$ Similar reactions have led also to ill-defined mixtures of acid-catalysed condensation products of bisnaphthalenes, believed to contain benzofluoranthenes. ${ }^{45 b}$ The $[j]$ and $[k]$ isomers are easily distinguished by melting point, UV spectra, colour and fluorescence. ${ }^{45 a}$ In the present work, only the $[j]$ isomer was formed with nitrobenzene as oxidant and at a much lower temperature than in the earlier work. This suggests that the acid-catalysed condensation followed by dehydrogenation did not proceed through the same mechanisms as those in the earlier work. ${ }^{45 a}$ The formation of only the $[j]$ isomer provides a credible mechanistic route (Scheme 2), whereby acid catalysed condensation of 1,2-dihydronaphthalene leads first to a specific dimer. The dimer can ring close in only one way to form a hydrobenzo[ $j]$ fluoranthene, which can be dehydrogenated to benzo[ $j]$ fluoranthene itself (Scheme 2). Confirmation of this pathway was obtained by isolation of a second benzo[j]fluoranthene, which was not completely identified but, from its UV and mass spectra, it is almost certainly a tetrahydronaphthyl-substituted benzo[ $j]$ fluroranthene (Scheme 2), formed by dehydrogenation of a trimer of 1,2-dihydronaphthalene. This tetrahydronaphthyl-susbstituted aromatic compound has an almost identical long wavelength UV absorption to that of benzo[ $j]$ fluoranthene but its molecular mass is 382 , rather than the 252 of a simple benzofluoranthene. The results suggest that the likelihood of any Scholl reactions being observed during nitrobenzene dehydrogenation requires first a sufficiently strong Lewis acid to induce condensation and then a nitrobenzene to effect dehydrogenation of this intermediate.

\section{(i) Effects of other acids}

Other acids were examined for their ability to accelerate dehydrogenation of DHA by nitrobenzene. All the experiments were carried out at $100{ }^{\circ} \mathrm{C}$ for 24 hours. For $\mathrm{HCl}$ (conc.), no conversion was found after 24 hours. However, when the reaction was left for over a week, a maximum $30 \%$ conversion was observed after 6 days. With acetic, trichloroacetic and trifluoroacetic acid, no reaction was observed. For concentrated nitric, sulfuric and chlorosulfonic acid, reaction was complete. The last acids are very strong and nitric acid is an oxidant in its own right. Compared with these acids, acetic, trichloroacetic and trifluoroacetic acids are quite weak and they seem not to be able to form an adequate complex with nitrobenzene. The reason for the failure of hydrochloric acid is not obvious except that, at $100{ }^{\circ} \mathrm{C}$, hydrochloric acid would evaporate from the reaction medium quite quickly. Also, it is notable that, whereas concentrated sulfuric acid has been reported to form a yellow solution with nitrobenzene, no colour developed in liquid $\mathrm{HCl}^{24 b}$

\section{(k) Reduction products of nitrobenzene}

When nitrobenzene is reduced electrochemically, two distinct patterns of final reduction products are observed. Under acidic conditions, nitrobenzene gives aniline and 4-hydroxyaniline (by acid-catalysed rearrangement of the intermediate reduction product, phenylhydroxylamine). At low $\mathrm{pH}$, the addition of a first electron is rate determining, followed by addition of a proton and then the rapid addition of three more electrons and protons to reach the phenylhydroxylamine stage, which then rearranges. Reduction to aniline requires another two electrons and two protons at a slightly higher reduction potential. With dehydrogenation in the presence of benzenesulfonic acid, the benzenesulfonate ester of 4-hydroxyaniline is observed. ${ }^{16}$ The dehydrogenations described here always resulted in the formation of aniline and the trifluoromethanesulfonate ester of 4-hydroxyaniline, This sulfonate has been synthesised earlier. ${ }^{10}$ Under alkaline conditions, proton addition becomes the rate controlling step and condensation of nitrosobenzene with aniline or phenylhydroxylamine to give azo and azoxy compounds is common. Thus, the reduction products of nitrobenzene (aniline and 4-aminophenyl trifluoromethylsulfonate) in the dehydrogenations described here are of the same pattern as those observed during electrochemical reduction under strongly acidic conditions. 


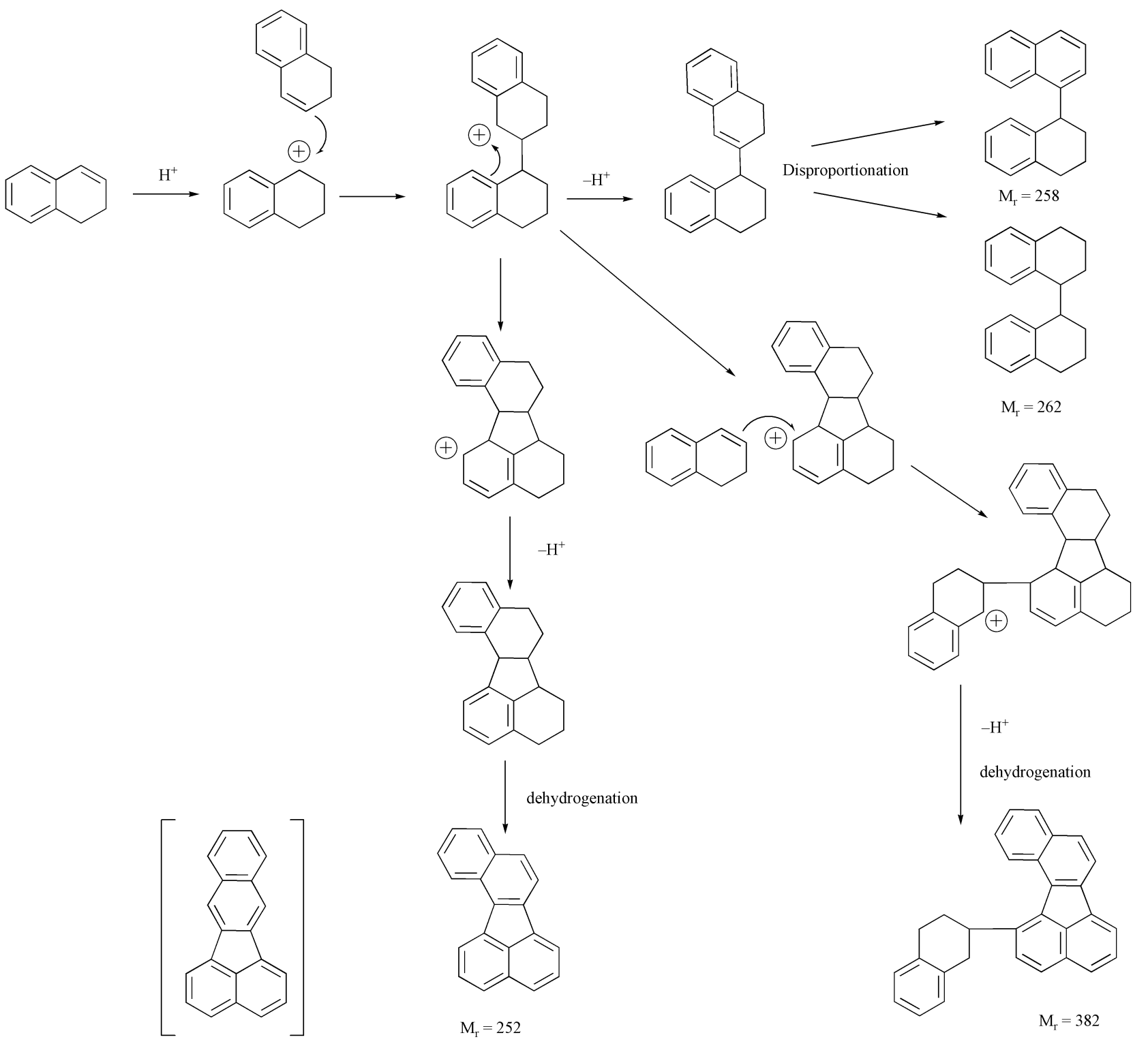

benzo[k]fluoranthene

benzo[j]fluoranthene

tetrahydronaphthylbenzo[j]fluoranthene

Scheme 2

\section{(I) Effect of aniline on the reaction rate for dehydrogenation of DHA}

In burst kinetics, after an initial burst phase, the subsequent steady state is controlled by one of the products of reaction. ${ }^{46}$ In the dehydrogenation of DHA by protonated nitrobenzene, it was found in the present work that intentional addition of anthracene at the beginning of reaction did not affect the burst or steady state phases. The other major reaction products are aniline and/or 4-aminophenol, which react with protons to form ammonium salts. The production of ammonium salts means that one product of reaction remains "complexed" to the catalyst (the acid), viz., the requirements for burst kinetics are met. ${ }^{46}$ As revealed by Fig. 1, with sufficient acid (more than equimolar to DHA), the burst in formation of anthracene is virtually quantitative within $2-3$ minutes at $100{ }^{\circ} \mathrm{C}$ (Fig. 1) and there was no steady state; in this case, the formation of ammonium salts is not sufficient to slow down anthracene formation. For amounts of acid less than equimolar to DHA, as acidity decreases the burst formation of anthracene becomes progressively less and longer steady state processes are observed. The data show that, after the burst phase, the resulting ammonium salt must control the subsequent steady state phase through control of hydrogen ion concentration; the concentration of hydrogen ions is controlled by dissociation of the
3. DHA $+\mathrm{PhNO}_{2}+6 \mathrm{e}^{-}+6 \mathrm{H}^{+}+\mathrm{n} \cdot \mathrm{H}^{+} \longrightarrow 3 . \mathrm{A}+\mathrm{PhNH}_{3}{ }^{+}+2 \mathrm{H}_{2} \mathrm{O}+(\mathrm{n}-1) \cdot \mathrm{H}$

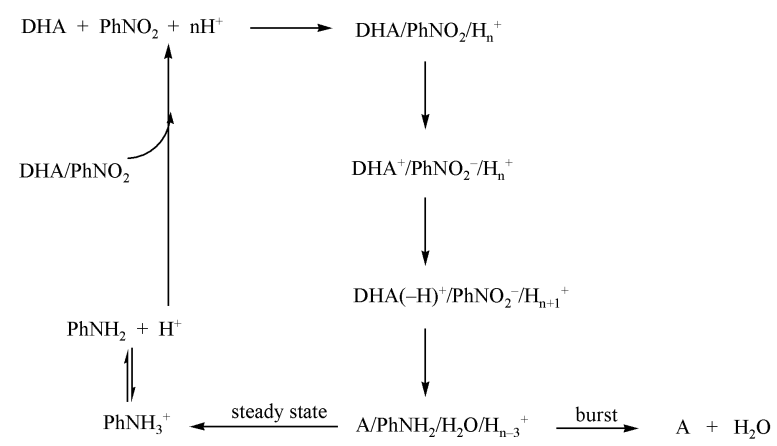

Scheme 3

anilinium salt. The process is illustrated in Scheme 3, in which the overall stoichiometric process is shown first and then the burst and steady state phases are outlined.

Further support for the process shown in Scheme 3 comes from two observations. First, in a system of nitrobenzene-acidDHA in which dehydrogenation is proceeding quite rapidly to give anthracene plus aniline, addition of a small molar excess of 
aniline to a reacting DHA-nitrobenzene-acid solution stops the reaction immediately because the additional aniline removes most of the protons. Second, when nitrobenzene is replaced as oxidant by 4-trifluoromethylnitrobenzene, rate changes are observed. For this last oxidant, formation of an ammonium salt from any 4-trifluoromethylaniline produced on reduction is much less easy than for aniline because the strongly electron-withdrawing $\mathrm{CF}_{3}$ group makes this aniline a very weak base. This means that one of the products of reaction does not remove so many protons and therefore the steady state rate is improved. Additionally, 4-trifluoromethylnitrobenzene has a lower reduction potential than does nitrobenzene. Therefore, during dehydrogenation of DHA, the electron-withdrawing $\mathrm{CF}_{3}$ substituent eases electron transfer and therefore speeds the burst phase. Both these effects (an increased burst and an increased steady state rate) were observed when DHA was dehydrogenated with 4-trifluoromethylnitrobenzene and trifluoromethanesulfonic acid at $100{ }^{\circ} \mathrm{C}$. The reduction products of 4-trifluoronitrobenzene were 4-trifluoromethylaniline and 2-amino-5-trifluoromethylphenyl trifluoromethanesulfonate.

\section{Conclusions}

Rate measurements and other experiments have shown that 9,10-dihydroanthracene is dehydrogenated to form anthracene by protonated nitrobenzenes in a process of burst kinetics. The results can be interpreted as initial complex formation between protonated nitrobenzene and dihydroanthracene, followed by electron and proton transfers from the DHA to the nitrobenzene. The action of trifluoromethanesulfonic acid is to increase the electron affinity (reduce the reduction potential) of the nitrobenzene through its protonation. The kinetics of dehydrogenation are very dependent on acid concentration, in keeping with the increased ease of electrochemical reduction of nitrobenzene in acidic media. A similar decrease in reduction potential can be effected through use of $p$-substituted nitrobenzenes, in which the substituent is electron-withdrawing.

The results provide a guide to the ease with which hydroarenes can be expected to be dehydrogenated to arenes with protonated nitrobenzenes. The major influence lies in the ionization energy $(I)$ of the substance to be dehydrogenated and the electron affinity $(E A)$ of the nitrobenzene used as oxidant, viz., on the difference $I-E A$. Since electron affinity varies over only a small range $(0-3 \mathrm{eV})$ but ionization energies for hydrocarbons vary over a much larger range $(0-10 \mathrm{eV})$, changing the ionization energy of the substrate to be dehydrogenated has a more substantial impact on the ease of dehydrogenation. The lower the ionization energy of the substrate, the easier it is to effect its dehydrogenation and vice versa. Experiments with $p$-substituted nitrobenzenes reveal that electron-withdrawing substituents accelerate the rate of dehydrogenation of porphyrinogens to porphyrins. The presence of the strong acid can lead to Friedel-Crafts type reactions and these in turn furnish compounds other than those expected of simple dehydrogenation. For example, 1,2-dihydronaphthalene forms little naphthalene on attempted dehydrogenation but does form a relatively high yield of benzo[ $j]$ fluoranthene by condensation and dehydrogenation.

The same criterion of the magnitude of $I-E A$ provides an explanation for the ease with which anions and radicals can be dehydrogenated by nitrobenzenes under neutral conditions. For anions and radicals in neutral or alkaline solution, their ionization energies are very much less than those of hydroarenes and the electron affinity of nitrobenzene decreases by only about 2 $\mathrm{eV}$. Therefore, $I-E A$ is very much less than it is in the case of protonated nitrobenzene and a hydroarene. Dehydrogenation of anions and radicals by nitrobenzene is expected to proceed easily, as is observed for many reactions, including SET processes.

\section{Experimental}

All compounds were used as received (Aldrich). GC analyses were carried out on either a Shimadzu GC14B instrument and an EC1 column or on a Chrompack CP-9001 with an Optima-5 column (FID). GC/MS analyses were carried out with an EC1 column on a Fisons Trio 1000 (EI at $70 \mathrm{~V}$ or CI with ammonia reagent gas).

\section{Kinetic experiments on the dehydrogenation of 9,10-dihydro- anthracene.}

(a) Initial experiments showing the burst phase (Fig. 1). In a typical experiment, 9,10-dihydroanthracene $(0.2 \mathrm{~g} ; 0.0011 \mathrm{~mol})$ was dissolved in nitrobenzene $(2 \mathrm{~mL})$ and trifluoromethanesulfonic acid $(x \mu \mathrm{L})$ was added. The mixture was placed in an oil bath at $373 \mathrm{~K}$ and small aliquots were removed at approximately $15 \mathrm{~min}$ intervals over a period of about $120 \mathrm{~min}$. These aliquots were examined by GC and the peak areas for anthracene (A) and dihydroanthracene (DHA) were measured. The conversion $(c=\mathrm{A} /(\mathrm{A}+\mathrm{DHA}))$ into anthracene was plotted against time to give the graphs shown in Fig. 1. The experiments were repeated for $x=10,20,30,40,50,150$.

(b) More accurate measurements over both burst and steady state phases. In a typical experiment, 9,10-dihydroanthracene $(0.2 \mathrm{~g} ; 1.11 \mathrm{mmol})$ was dissolved in nitrobenzene $(2 \mathrm{~mL})$. Trifluoromethanesulfonic acid $(x \mu \mathrm{L})$ was added and the final mixture was stirred in an oil bath stabilised at $373.15 \pm 1 \mathrm{~K}$. The temperature was noted throughout the kinetic runs to monitor its constancy. Small aliquots $(50 \mu \mathrm{L})$ of the reaction mixture were removed at regular intervals and each sample was added to dichloromethane $(1 \mathrm{~mL})$ ready for $\mathrm{GC}$, which was used to monitor the formation of anthracene and the disappearance of 9,10-dihydroanthracene. For each sample, peak areas for both anthracene (A) and 9,10-dihydroanthracene (DHA) were measured. A dimensionless conversion factor $(c)$ was calculated from, $c=\mathrm{A} /(\mathrm{A}+\mathrm{DHA})$. Rate constants were then determined as described in the Discussion section. This kinetic experiment was repeated at two more temperatures, $353.15 \pm 1$ and $393.15 \pm 1 \mathrm{~K}$.

For each of the three temperatures, rate constants were determined at acidities corresponding to the addition of $x \mathrm{~mL}$ of trifluoromethanesulfonic acid, where $x=10,20,30,40$, and 50 .

Each rate constant was measured in duplicate from 6 to 8 data points, giving a coefficient of variation between sets of $2.4 \%$. The rate constants are listed in Table 1 .

\section{Kinetic experiments on the dehydrogenation of porphyrinogens.}

(a) Preparation of 5,10,15,20-tetrakis(4-methoxyphenyl)porphyrinogen. 5,10,15,20-Tetrakis(4-methoxyphenyl)porphyrin $(0.5 \mathrm{~g} ; 0.68 \mathrm{mmol})$ was dissolved in a mixture of glacial acetic acid $(50 \mathrm{~mL})$ and trifluoroacetic acid $(2.5 \mathrm{~mL})$ and the resulting solution was degassed by refluxing it gently under a slow stream of nitrogen. After cooling the solution to $20^{\circ} \mathrm{C}$, zinc dust $(2.5 \mathrm{~g}$; $0.38 \mathrm{~mol})$ was added and the solution was stirred for $1 \mathrm{~h}^{47}$ To ensure complete reduction, visible spectra of small aliquots were obtained until no bands could be observed in the $400-600 \mathrm{~nm}$ region. This solution of porphyrinogen (S) was filtered and used in the following kinetic work.

(b) Dehydrogenation back to porphyrin. In a typical experiment, two vessels were used, which could be heated in the same oil bath and the contents of which could be stirred. Nitrogen was used to ensure the atmospheres within the vessels contained no air. Into one vessel was placed an aliquot $(10 \mathrm{~mL})$ of the porphyrinogen solution (S), which served as a "blank" control. Into the other vessel was placed an aliquot of solution (S) plus 
nitrobenzene $(1.5 \mathrm{mmol})$. Both vessels were heated to $363 \pm 1 \mathrm{~K}$ and stirring was initiated at zero time. Aliquots $(25 \mu \mathrm{L})$ of each solution were removed at intervals and each was dissolved in $\mathrm{CHCl}_{3}(3 \mathrm{~mL}$, containing $13 \mathrm{~mL}$ of triethylamine per litre of $\mathrm{CHCl}_{3}$ ). Visible spectra were recorded and absorbances due to the appearance of 5,10,15, 20-tetrakis(4-methoxyphenyl)porphyrin were measured at $420 \mathrm{~nm}$ (the Soret band). The background absorbance of the "blank" at $420 \mathrm{~nm}$ was subtracted from the measured absorbances from the reaction vessel. Concentrations of porphyrin were calculated from the net absorbance and the molar absorption coefficient for the Soret band of the porphyrin. ${ }^{48}$ The initial rate of formation of porphyrin was determined from its yield over the first few minutes of reaction. The experiment was repeated for other substituted nitrobenzenes $\left(4-\mathrm{RC}_{6} \mathrm{H}_{4} \mathrm{NO}_{2} ; \mathrm{R}=\mathrm{NO}_{2}, \mathrm{NH}_{3}{ }^{+}\right.$, $\left.\mathrm{N}\left(\mathrm{CH}_{3}\right)_{3}{ }^{+}, \mathrm{CH}_{3} \mathrm{O}, \mathrm{CO}_{2} \mathrm{H}\right)$ in place of nitrobenzene itself $(\mathrm{R}=$ $\mathrm{H})$. Logarithms of the initial rates of formation of porphyrin for the various nitrobenzenes are shown in Fig. 3.

\section{Dehydrogenation of 9,10-dihydroanthracene by other acids}

Each experiment was carried out using a solution of 9,10-dihydroanthracene $(0.2 \mathrm{~g} ; 1.11 \mathrm{mmol})$ in nitrobenzene $(2 \mathrm{~mL})$, to which had been added an acid $(50 \mu \mathrm{L})$. Each solution was heated at $373.15 \pm 1 \mathrm{~K}$ for $24 \mathrm{~h}$ and the amount of conversion to anthracene was determined by gas chromatography, as described above. The acids used were either concentrated or neat: $\mathrm{HCl}$ (conc.), $\mathrm{HNO}_{3}$ (conc.), $\mathrm{H}_{2} \mathrm{SO}_{4}$ (conc.), $\mathrm{HSO}_{3} \mathrm{Cl}$ (conc.), $\mathrm{CH}_{3} \mathrm{CO}_{2} \mathrm{H}, \mathrm{CF}_{3} \mathrm{CO}_{2} \mathrm{H}, \mathrm{CCl}_{3} \mathrm{CO}_{2} \mathrm{H}$. The results are described in the main text.

\section{Dehydrogenation of other hydrocarbons}

(a) 1,2-Dihydronaphthalene (dialin). The hydrocarbon $(0.14 \mathrm{~g}$; $1.1 \mathrm{mmol})$ was dissolved in nitrobenzene $(2 \mathrm{~mL})$ and trifluoromethanesulfonic acid $(30 \mu \mathrm{L})$ was added. The mixture was kept at $295 \pm 1 \mathrm{~K}$ and was sampled at intervals for GC and GC/MS analysis. Naphthalene was formed quickly and starting material disappeared within a few minutes but the conversion to naphthalene was very low. The experiment was repeated at $373 \mathrm{~K}$ and $423 \mathrm{~K}$. Results are described in the main text. After the experiment at $423 \mathrm{~K}$, some of the product was separated on silica gel TLC plates to give mainly one yellow band, along with two other smaller bands. The bands were scraped from the plate and extracted with DCM. The isolated products were examined by GC/MS. For the middle-running main yellow band, the product crystallised on evaporation of the solvent and was identified as benzo[j]fluoranthene $\left(\mathrm{mp} 164{ }^{\circ} \mathrm{C}\right.$, lit. $166{ }^{\circ} \mathrm{C} ; ;^{45 a}$ UV/visible $\lambda_{\max } 332,346,364,374,382 \mathrm{~nm}$; MS (EI), $m / z 252$ $\left(\mathrm{M}^{+} ; 100 \%\right), 250\left(\mathrm{M}-\mathrm{H}_{2} ; 36 \%\right), 126\left(\mathrm{M}-\mathrm{C}_{10} \mathrm{H}_{6} ; 30 \%\right)$, $125\left(\mathrm{M}-\mathrm{C}_{10} \mathrm{H}_{7} ; 30 \%\right)$. A bottom paler yellow band was tentatively identified as a naphthylbenzo[ $j]$ fluoranthene (UV/visible $\lambda_{\max } 332,338,346,366,374,386 \mathrm{~nm} ; \mathrm{MS}\left(\mathrm{CI} ; \mathrm{NH}_{4}^{+}\right), \mathrm{m} / z 383$ $\left(\mathrm{M}+\mathrm{H}^{+} ; 100 \%\right), 131\left(\mathrm{M}-\mathrm{C}_{10} \mathrm{H}_{11} ; 22 \%\right)$. The top weakly fluorescent band produced the same mass spectrum as that found for 1,2-dihydronaphthalene "dimers", observed in the initial GC/MS analysis. Results are discussed in greater detail in the main text. In the following, experimental conditions were similar to those in (a) at $295 \mathrm{~K}$.

(b) 1,2,3,4-tetrahydronaphthalene (tetralin). This showed no conversion to dehydrogenated products when heated at $423 \mathrm{~K}$ for $60 \mathrm{~min}$.

(c) Cyclohexene. At $323 \mathrm{~K}$, conversion to benzene was $40 \%$ complete after $1 \mathrm{~h}$.

(d) Cyclohexadiene. Complete conversion to benzene was observed within 1 minute (GC and GC/MS).

(e) 1-Methyl-4-isopropylcyclohexa-1,3-diene ( $\alpha$-terpinene). Complete conversion to $p$-cymene (1-methyl-4-isopropenylbenzene) was observed in $5 \mathrm{~min}$. (f) $(4-R)(+)$ and $(S)(-)$-isopropenyl-1-methylcyclohexene (limonenes). Complete conversion to 1-methy-4-isopropenylbenzene was observed within $12 \mathrm{~min}$.

(g) Cyclohexane. No change was observed at $323 \mathrm{~K}$ after extended periods $(24 \mathrm{~h})$.

\section{Formation of reduction products from nitrobenzene}

In all experiments using nitrobenzene as dehydrogenating agent, its reduction product, aniline, was observed and confirmed by GC/MS. Additionally, a second reduction product was found (4-aminophenoxytrifluoromethyl sulfonate; MS, $\mathrm{m} / \mathrm{z}$ $241\left(\mathrm{M}^{+}, 8 \%\right), 108\left(\mathrm{M}-\mathrm{CF}_{3} \mathrm{SO}_{2} ; 100 \%\right), 80\left(\mathrm{M}-\mathrm{CF}_{3} \mathrm{SO}_{2}-\right.$ $\mathrm{CO} ; 45 \%)$. The identity of this product of acid catalysed rearrangement of initially formed phenylhydroxylamine was confirmed by independent synthesis. ${ }^{10}$ When 4-trifluoromethylnitrobenzene was used as dehydrogenating agent, the analogous reduction product (2-amino-5-trifluoromethylphenoxytrifluoromethyl sulfonate) was observed; $\mathrm{MS},\left(\mathrm{M}^{+}, \mathrm{m} / \mathrm{z} 309 ; 10 \%\right), 176$ $\left(\mathrm{M}-\mathrm{CF}_{3} \mathrm{SO}_{2} ; 100 \%\right), 148\left(\mathrm{M}-\mathrm{CF}_{3} \mathrm{SO}_{2}-\mathrm{CO} ; 85 \%\right)$.

\section{Complex formation between protonated nitrobenzene and 9,10-dihydroanthracene}

A solution ( $\mathrm{S} 1)$ of 9,10-dihydroanthracene in nitrobenzene $\left(0.29 \mathrm{~mol} \mathrm{~L}^{-1}\right)$ and a solution (S2) of trifluoromethanesulfonic acid in nitrobenzene $\left(1.28 \mathrm{~mol} \mathrm{~L}^{-1}\right)$ were prepared. To aliquots $(5.2 \mathrm{~mL})$ of S1 were added aliquots of S2 $(200 \mu \mathrm{L})$ to a limit of $1000 \mu \mathrm{L}$. UV/visible spectra for each mix were recorded and compared with a blank solution of S1 containing no acid. A plot of net absorbance at $520 \mathrm{~nm}$ against amount of added acid was linear, showing that the Beer-Lambert laws were obeyed. As more acid was added, the absorbance of nitrobenzene near $470 \mathrm{~nm}$ increased until, after addition of $600 \mu \mathrm{L}$ of acid solution S2, a clear maximum of the charge-transfer band could be discerned. The tail of this band at $520 \mathrm{~nm}$ was used for the absorbance measurements. Finally, the logarithm of the absorbance at $520 \mathrm{~nm}$ was plotted against the logarithm of the hydrogen ion concentration and gave a straight line of slope 1.81 (correlation coefficient, 0.9917).

\section{Acknowledgements}

The authors thank FCT, Portugal (JMTBV, DJPG, MLSC) and the Eschenmoser Trust, UK (MLSC) for generous financial assistance.

\section{References}

1 (a) P. A. Claret, in Comprehensive Organic Chemistry, eds. D. H. R. Barton and W. D. Ollis, vol. 4, Pergamon Press, Oxford, 1979, pp. 157-160; (b) L. Golser, in Methoden der Organischen Chemie (Houben-Weyl), eds. E. Müller and O. Bayer, Oxidation Part 4/1b; Part II, Oxidation, Georg Thieme, Stuttgart, 1975, pp. 963; (c) F. G. Holliman, B. A. Jeffrey and D. J. H. Brock, Tetrahedron, 1963, 19, 1841; Y. Lepage, Bull.Soc. Chim. Fr., 1963, 1141; (d) H. T. Clarke and A. W. Davis, in Organic Syntheses, collected vol. 1, Wiley, London, 1948, p. 478; S. Filipov, O. Petrov and N. Mollov, Arch. Pharm., $1982,315,498$.

2 M. Okawara and K. Yamamoto, Kegyo Kagaku Zasschi, 1957, 60, 78 (Chem. Abs., 1959, 53, 6179h).

3 (a) K. G. Allum, I. V. Howell and R. C. Pitkethly, Brit. Pat., 1,378,151, 1974, (Chem. Abstr., 1975, 82, 125043r); (b) N. N. Rozhdestvenskaya, G. V. Isagulyanto, L. A. Kupcha and N. S. Kozlov, Izv. Akad. Nauk SSSR, Ser. Khim., 1991, 1751 (Chem. Abs., 1992, 116, 20722d); J. Haber, M. Wojciechowska and W. Gut, Bull. Pol. Acad. Sci. Chem., 1996, 44, 55 (Chem. Abs., 1996, 125, 301640a); N. S. Kozlov, K. Zhavnerlo and M. I. Kozlyak, Dokl. Akad. Nauk Beloruss SSR, 1970, 14, 914 (Chem. Abs., 1971, 74, 76099n); H. S. Kim, D. I. Kim, C-S. Kim and Y. J. Joo, Kongop Hwahak, 1994, 5, 871 (Chem. Abs., 1995, 123, 143142s). 
4 T. J. Wallace, J. M. Miller, H. Probner and A. Schriesheim, Proc. Chem. Soc., 1962, 384; T. J. Wallace, A. Schriesheim and W. Bartok, J. Org. Chem., 1963, 28, 1311.

5 G. A. Russel and E. G. Janzen, J. Am. Chem. Soc., 1962, 84, 4513; G. A. Russel, R. G. Janzen, H.-D. Becker and F. Smentowski, J. Am. Chem. Soc., 1962, 84, 2652

6 (a) R. D. Guthrie and N. S. Cho, J. Am. Chem. Soc., 1975, 97, 2280; (b) D. N. R. Rao and R. F. Mason, Basic Life Sci., 1988, 49, (Oxygen Radicals in Biology and Medicine, 787); M. Rahman and S. K. Naher, J. Organomet. Chem., 1987, 329, 133.

7 M. K. Eberhardt, Tetrahedron Lett., 1984, 25, 3663; J. Eibenberger, D. Schulte-Frohlinde and S. Steenben, J. Phys. Chem., 1980, 84, 704.

8 N. Kornblum and M. J. Fifolt, Tetrahedron, 1989, 45, 1311; L. Weisler and R. W. Helmkamp, J. Am. Chem. Soc., 1945, 67, 1167; R. Bacaloglu, A. Blasko, C. Bunton, E. Dorwin, F. Ortega and C. Zucco, J. Am. Chem. Soc., 1991, 113, 238.

9 M. Hudlicky, Reductions in Organic Synthesis, ACS Monograph 188, American Chemical Society, Washington DC, 1996, pp. 94-95.

10 R. P. Austin and J. H. Ridd, J. Chem. Soc., Chem. Commun., 1992 1599; R. P. Austin and J. H. Ridd, J. Chem. Soc., Perkin Trans. 2, 1994, 1411; R. P. Austin and J. H. Ridd, J. Chem. Soc., Perkin Trans. $2,1994,1205$

11 G. A. Clowes, J. Chem. Soc. (C), 1968, 2519.

12 I. D. Entwistle, R. A. W. Johnstone and A. H. Wilby, Chemical Reviews, 1985, 85, 129.

13 J. March, Advanced Organic Chemistry, 4th edn., Wiley, New York, 1992, pp. 1216-1217.

14 D. H. Geske and A. H. Maki, J. Am. Chem. Soc., 1960, 82, 2671; B. Kalyanaram, E. Perez-Reyes and R. P. Mason, Mol. Pharm., 1979, 16, 1059; B. Kalyanaram, R. P. Mason, R. Rowlett and L. D. Kispert, Biochim. Biophys. Acta, 1981, 660, 102; Y. Ito, T. Konoika and T. Saeguta, Terahedron Lett., 1974, 1287.

15 A. J. Fry, Synthetic Organic Electrochemistry, Harper and Row, New York, 1972, pp. 224-234; A.J. Fry, in The Chemistry of Amino, Nitroso and Nitro Compounds and their Derivatives, suppl. F, part 1 , ch. 8, ed. S. Patai, Wiley, Chichester, 1982, p. 319; B. Kastening, Electrochim. Acta, 1964, 9, 241

16 C. D. Nenitzescu and A. Balaban, Chem. Ber., 1958, 91, 2109.

17 A. J. Gordon and R. A. Ford, The Chemist's Companion, Wiley, New York, 1972, pp. 138-140.

18 All heats of formation are known accurately for a temperature of $25^{\circ} \mathrm{C}$ : dihydroanthracene $\left(167 \mathrm{~kJ} \mathrm{~mol}^{-1}\right),{ }^{19}$ anthracene $(234 \mathrm{~kJ}$ $\left.\mathrm{mol}^{-1}\right){ }^{19}$ nitrobenzene $\left(67 \mathrm{~kJ} \mathrm{~mol}^{-1}\right),{ }^{20 a}$ aniline $\left(88 \mathrm{~kJ} \mathrm{~mol}^{-1}\right){ }^{20 a}$ water $\left(-242 \mathrm{~kJ} \mathrm{~mol}^{-1}\right)^{20 a}$

19 A. Magnus, H. Hartman and F. Becker, Z. Physik. Chem., 1951, 197, 85.

20 Handbook of Chemistry and Physics, 76th edn., ed. D. R. Lide, CRC Press, Boca Raton, 1995; (a) pp. 5-36 and 5-16; (b) p. 6-170.

21 J. E. Leffler, Science, 1953, 117, 340; G. S. Hammond, J. Am. Chem. Soc., 1955, 77, 334.

22 L. J. Andrews, Chem. Rev., 1954, 54, 713; L. J. Andrews and R. M. Keefer, Molecular Complexes in Organic Chemistry, Holden-Day, San Francisco, 1964; H. McConnell, J. S. Haw and R. Platt, J. Phys. Chem., 1953, 21, 66.

$23 \mathrm{H}$. H. Jaffe and M. Orchin, Theory and Applications of Ultraviolet Spectroscopy, Wiley, New York, 1964; (a) pp. 578-585; (b) pp. 271273.

24 (a) O. I. Kachurin and V. M. Belobrov, Ukr. Khim. Zh., 1975, 41 709; E. M. Arnett, R. P. Quirk and J. J. Burke, J. Am. Chem. Soc., 1970, 92, 1260; R. C. Paul, K. C. Khanna and K. C. Malhotra, Res. Bull. Panjab Univ., 1965, 16, 251; R. J. Gillespie and E. A. Robinson, J. Chem. Soc., 1957, 4233; (b) M. E. Peach and T. C. Waddington, J. Chem. Soc., 1962, 2680.

25 H. C. Brown and M. Grayson, J. Am. Chem. Soc., 1953, 75, 6285.

26 H. Siegerman, in Technique of Electroorganic Synthesis, vol. V, part II, ed. N. L. Weinberg, Wiley, New York, 1975, pp. 667.

27 J. E. Page, J. W. Smith and J. G. Weller, J. Phys. Chem., 1949, 53, 545 S. K. Vijayalakshamma and R. S. Subrahmanya, J. Electroanal. Chem., 1969, 23, 99 .

28 J.O'M. Bockris and A. K. N. Reddy, Modern Electrochemistry, Plenum Press, New York, p. 906

29 R. S. Mulliken, J. Am. Chem. Soc., 1952, 74, 811.

30 The three terms are: the ionization energy $(I)$ for 9,10dihydroanthracene $(8.56 \mathrm{eV}),{ }^{31}$ the electron affinity $(E A)$ for protonated nitrobenzene $(2.6 \mathrm{eV}),{ }^{32}$ and the Coulombic energy $(C)$ of attraction between a positive and a negative ion $(0.3 \mathrm{eV}){ }^{33}$ The frequency $(v)$ for a charge-transfer band at $470 \mathrm{~nm}$ is predicted approximately by the equation, $h v=1+2 \beta^{2} /(I-E A+C) ; \beta$ is a resonance integral. ${ }^{23 b, 29}$ Using the value for $h v$ of $2.63 \mathrm{eV}$ for the $470 \mathrm{~nm}$ charge transfer, $I$ and $E A$ from the literature, and $C$ by calculation, a value of $1.81 \mathrm{eV}$ for $\beta$ can be derived, which lies within the reported small range of values for this integral. ${ }^{34}$ The resonance energy gained on forming the tight complex is given by $\beta^{2} /(I-E A+$ C) ${ }^{23 b, 29}$ which in the present case for DHA- $\mathrm{PhNO}_{2}-\mathrm{H}^{+}$is $78 \mathrm{~kJ}$ $\mathrm{mol}^{-1}$

31 In the absence of an ionization energy for DHA itself, the value for 1,2-dimethylbenzene was used. K. Watanabe, J. Chem. Phys., 1957, 26, 542 .

32 The electron affinity for protonated nitrobenzene is unknown but it can be estimated quite accurately. The largest electron affinities are about $3 \mathrm{eV}$ and nitrobenzene itself has a value of $1.01 \mathrm{eV}^{35}$ It might therefore be expected that protonated nitrobenzene should have an electron affinity in the range $1.01<E A<3.0 \mathrm{eV}$. It has been pointed out that reduction potentials $\left(E_{1 / 2}\right)$ should correlate with the orbital energy of the first unoccupied molecular orbital in a molecule. ${ }^{36}$ Electron affinity should also correlate with this same orbital energy and, therefore, there should be a linear correlation between electron affinity $(E A)$ and reduction potential $\left(E_{12}\right)$. By using values for electron affinities for a series of compounds ${ }^{35}$ and known $E_{1 / 2}$ reduction potentials for the same compounds, ${ }^{37}$ a graph can be plotted, which gives a good linear correlation with, $E A=1.49\left(E_{1 / 2}\right)$ $+2.79 \mathrm{eV}$ (correlation coefficient $=0.9847$ ). At the acidities used in the experiments described here, the $E_{1 / 2}$ value for nitrobenzene is $-0.14 \mathrm{~V}^{27}$ Therefore, from the linear correlation with $E A$, the electron affinity of protonated nitrobenzene is estimated to be 2.6 $\mathrm{eV}$.

33 The Coulombic term $(C)$ or potential energy for the complex can be derived from the expression, $C=z_{1} z_{2} \mathrm{~N}_{\mathrm{A}} e^{2} / 4 \pi \varepsilon_{0} r$, in which $z_{1}, z_{2}$ are the numbers of charges $\left(z_{1}=z_{2}=1\right.$ for transfer of one electron), $\mathrm{N}_{\mathrm{A}}$ is Avagadro's number, $e$ is the charge on the electron $\left(1.6 \times 10^{-19} \mathrm{C}\right)$, $\varepsilon_{0}$ is the permittivity of the solution (= permittivity of free space $\times$ dielectric constant for nitrobenzene $\left.=3.19 \times 10^{-10} \mathrm{~F} \mathrm{~m}^{-1}\right)$ and $r$ is the distance apart of the two charged species (closest approach of two carbon atom radii $\left(1.34 \times 10^{-10} \mathrm{~m}\right)$. Insertion of these values gives $C$ $=0.3 \mathrm{eV}$.

34 R. L. Flurry, Molecular Orbital Theories of Bonding in Organic Molecules, Edward Arnold, London, 1968, p. 59.

35 S. Chowdhury, T. Heinis, E. Grimsrud and P. Kebarle, J. Phys. Chem., 1986, 90, 2747

36 G. J. Hoijtink, Rec. Trav. Chim. Pays-Bas, 1955, 1525; G. Cauquis, in Organic Electrochemistry, ed. M. M. Baizer, Marcel Dekker, New York, 1973, pp. 75-79.

37 Reduction potentials were taken from reference 26, (pp. 752, 769, $770,920,934-938)$. Fourteen compounds were found to be common to references 26,35 and these were used for the correlation of $E_{1 / 2}$ with $E A$. Values for $E_{1 / 2}$ from reference 26 were those relating to solutions in $\mathrm{MeCN}$ at $\mathrm{pH} 7$, with a $\mathrm{Hg}$ electrode and a standard calomel electrode as reference.

38 F. H. Herbstein, M. Kapon and G. M. Reisner, Acta Crystallogr. Sect. $B, 1986, \mathbf{4 2}, 181$.

39 L. R. Melby, R. J. Harder, W. R. Hertler, W. Mahler, R. E. Benson and W. E. Mochel, J. Am. Chem. Soc., 1962, 84, 3374

40 The heat capacity for nitrobenzene is $186 \mathrm{~J} \mathrm{~mol}^{-1} \mathrm{~K}^{-1}{ }^{20 b}$ Heat content per mole of nitrobenzene heated from 20 to $100{ }^{\circ} \mathrm{C}=186 \times$ $80=15 \mathrm{~kJ} \mathrm{~mol}^{-1}$. This is a minimum value because, in the experiments described here, the nitrobenzene was always used in excess as a solvent as well as a reactant.

41 J. L. Franklin, J. G. Dillard, H. M. Rosenstock, J. T. Herron, K. Draxl and F. H. Field, Ionization Potentials, Appearance Potentials, and Heats of Formation of Gaseous Positive Ions, National Bureau of Standards, NSRDS-NBS 26, Washington DC, 1969 , cyclohexane $9.86 \mathrm{eV}$, cyclohexene $8.95 \mathrm{eV}$, cyclohexadiene $8.4 \mathrm{eV}$.

42 Y. Zhao and F. G. Bordwell, J. Org. Chem., 1996, 61, 2530.

43 C. Hansch, A. Leo and R. W. Taft, Chem. Rev., 1991, 91, 185.

44 R. A. W. Johnstone, M. L. P. G. Nunes, M. M. Pereira, A. M. d'A Rocha Gonsalves and A. Serra, Heterocycles, 1996, 42, 1423 and references cited therein.

45 (a) M. Orchin and L. Reggel, J. Am. Chem. Soc., 1947, 69, 505; (b) C. D. Nenitzescu and M. Auram, J. Am. Chem. Soc., 1950, 72, 3486; A. Dansi and M. Reggiani, Gazz. Chim. Ital., 1948, 78, 801

46 W. P. Jenks, Catalysis in Chemistry and Enzymology, McGraw-Hill, New York, 1969; A. Fersht, Enzyme Structure and Mechanism, W. H. Freeman, London, 1979.

47 D. Dolphin, J. Heterocycl. Chem., 1970, 7, 275.

48 D. W. Thomas and A. E. Martell, J. Am. Chem. Soc., 1956, 78, 1335. 